Comment. Math. Helv. 74 (1999) 507-529

(C) 1999 Birkhäuser Verlag, Basel

$0010-2571 / 99 / 040507-23 \$ 1.50+0.20 / 0$

Commentarii Mathematici Helvetici

\title{
Gradient flows within plane fields
}

\author{
John Etnyre and Robert Ghrist
}

\begin{abstract}
We consider the dynamics of vector fields on three-manifolds which are constrained to lie within a plane field, such as occurs in nonholonomic dynamics. On compact manifolds, such vector fields force dynamics beyond that of a gradient flow, except in cases where the underlying manifold is topologically simple (i.e., a graph-manifold). Furthermore, there are strong restrictions on the types of gradient flows realized within plane fields: such flows lie on the boundary of the space of nonsingular Morse-Smale flows. This relationship translates to knot-theoretic obstructions for the link of singularities in the flow. In the case of an integrable plane field, the restrictions are even finer, forcing taut foliations on surface bundles. The situation is completely different in the case of contact plane fields, however: it is easy to realize gradient fields within overtwisted contact structures (the nonintegrable analogue of a foliation with Reeb components).
\end{abstract}

Mathematics Subject Classification (1991). 34C35, 58A30, 57M25, 57R30.

Keywords. Gradient flows, foliations, contact structures, round handles, knots.

\section{Introduction}

Let $M$ denote a compact oriented three-manifold. A plane field $\eta$ on $M$ is a subbundle of the tangent bundle $T M$ which associates smoothly to each point $p \in M$ a two-dimensional subspace $\eta(p) \subset T_{p} M$. Unlike line fields, a plane field cannot always be integrated to yield a two-dimensional foliation $\mathcal{F}$. A plane field is said to be integrable if it can be "patched together" to yield a foliation whose leaves are tangent to the plane field at each point. Certainly, such plane fields have strong topological and geometric properties. On the other hand, the case where the plane field $\eta$ is nowhere integrable can be equally important. A maximally nonintegrable (in the sense of Frobenius - see $\$ 5$ ) plane field on an odd-dimensional manifold is a contact structure. Seen as an "anti-foliation", contact structures are rich in geometric and topological properties which of late have become quite important in understanding the topology of three-manifolds and the symplectic geometry of four-manifolds.

Let $X$ be a vector field on $M$. The dynamics of $X$ are often related to global properties of $M$. If we further specify that $X$ is tangent to a plane field $\eta$ - that 
is, $X(p) \in \eta(p)$ for all $p \in M$ - then we might expect stronger relationships. We will consider the ways in which the topology and geometry of a plane field $\eta$ are coupled to the dynamics of vector fields contained in $\eta$. The general principal at work here as elsewhere is that simple dynamics implicate simple topological objects in dimension three. We will reassert this by examining the gradient flows within plane fields.

The examination and classification of gradient flows has been ubiquitous in the study of manifolds: e.g., the h-cobordism theorem and the resolution of the high-dimensional Poincaré Conjecture. This paper will add to the typical scenario the constraint of lying within a plane field. Atypical restrictions on the dynamics and on the underlying manifold are born out of this.

We note that the problem of understanding gradient fields constrained to lie within plane fields is by no means unnatural. The study of mechanical systems with nonholonomic constraints is precisely the study of flows constrained to lie within a nowhere integrable distribution (i.e., in odd dimensions, a contact structure). For example, gradient flows for mechanical systems have been used successfully in the control of robotic systems (see, e.g., [15]): to maneuver a robot from points $A$ to $B$ through a physical space replete with obstacles, one establishes a gradient flow on a suitable configuration space with $B$ as a sink, with $A$ in the basin of attraction for $B$, and with infinite walls along the obstacles. In this paper, we show that the nonholonomic version of this procedure possesses potentially difficult topological obstructions.

The paper is organized as follows: the remainder of this section provides a brief sketch of the requisite theory from the dynamical systems approach to flows. In $\S 2$, we commence our investigation of plane field flows by examining local and global properties of fixed points: fixed points will not be isolated, but must (on an open dense subset of $C^{r}$ vector fields tangent to $\eta, r \geq 1$ ) rather appear in links, or embedded closed curves. This culminates in a classification of gradient flows on three-manifolds which can lie within a plane field in $\S 3$. The existence of such flows is equivalent to the existence of a certain type of round handle decomposition for the manifold (see Definition 3.2). Surprisingly, this same restriction appears when considering energy surfaces for (Bott-) integrable Hamiltonian flows [3].

Theorem. Let $M$ be a compact 3-manifold outfitted with a plane field $\eta$. If $X$ is a nondegenerate ${ }^{1}$ gradient field tangent to $\eta$, then $X$ lies in the boundary of the space of nonsingular Morse-Smale flows on $M$. Furthermore, the set of fixed points for $X$ forms the cores of an essential round handle decomposition for $M$.

This leads to the corollary (a stronger form of which is proved in $\S 3$ ):

Corollary. Non-gradient dynamics is a generic (residual) property in the class of

1 See Definition 2.9. 
$C^{r}(r \geq 4)$ vector fields tangent to a fixed $C^{r}$ plane field on a closed hyperbolic three-manifold.

In $\S 4$, we consider the manifestation of these restrictions on a knot-theoretic level for the particular case of the 3 -sphere.

Theorem. For $X$ a nondegenerate gradient plane field flow on $S^{3}$, each connected component of the fixed point set of $X$ is a knot whose knot type is among the class generated from the unknot by the operations of iterated cabling and connected sum.

We proceed with remarks on two cases in which the plane field carries additional geometric structure: first, the case of an everywhere integrable plane field, i.e., a foliation; and second, the case of a maximally nonintegrable plane field, i.e., a contact structure. The property of carrying a gradient flow in a foliation forces the foliation to be taut; hence, there are no (nondegenerate) gradient flows within a foliation on $S^{3}$. More generally, we have the following restrictions on the underlying three-manifold:

Theorem. A closed orientable three-manifold containing a nondegenerate gradient field within a $C^{r}(r \geq 2)$ codimension-one foliation must be a surface bundle over $S^{1}$ with periodic (or reducibly periodic) monodromy map.

The corresponding restrictions do not hold for the contact case. We demonstrate that gradient fields can always reside within the analogue of a non-taut foliation: an overtwisted contact structure. We close with two questions on the higher dimensional versions of the results of this paper.

\subsection{The dynamics of flows}

Ostensibly, flows within a plane field would appear to be a relatively restricted class of objects. However, the dynamics of such flows can exhibit behaviors which range from strictly two-dimensional dynamics (as when the plane field yields a foliation by compact leaves) to fully three-dimensional phenomena (e.g., an Anosov flow, which is tangent to a pair of transverse integrable plane fields). In $\S 2$, we show that near a fixed point of a plane field flow, the dynamics are locally "stacked" planar dynamics. In contrast, it is a simple exercise in homotopy theory that every nonsingular flow on $S^{3}$ (or any integral homology 3-sphere) lies within a plane field.

A few definitions are important for the dynamical systems theory used in this paper. The most important aspect of a flow with respect to its geometry and dynamics is the notion of hyperbolicity. Recall that an invariant set $\Lambda \subset M$ of a flow $\phi^{t}$ is hyperbolic if the tangent bundle $\left.T M\right|_{\Lambda}$ has a continuous $\phi^{t}$-invariant splitting into $E^{\phi} \oplus E^{s} \oplus E^{u}$, where $E^{\phi}$ is tangent to the flow direction, and $D \phi^{t}$ uniformly contracts and expands along $E^{s}$ and $E^{u}$ respectively: i.e., 


$$
\begin{aligned}
\left\|D \phi^{t}\left(\mathbf{v}^{s}\right)\right\| & \leq C e^{-\lambda t}\left\|\mathbf{v}^{s}\right\| \text { for } \mathbf{v}^{s} \in E^{s} \\
\left\|D \phi^{-t}\left(\mathbf{v}^{u}\right)\right\| & \leq C e^{-\lambda t}\left\|\mathbf{v}^{u}\right\| \text { for } \mathbf{v}^{u} \in E^{u}, t>0,
\end{aligned}
$$

for some $C \geq 1$ and $\lambda>0$. A flow $\phi^{t}$ which is hyperbolic on all of $M$ is called an Anosov flow.

The existence of hyperbolic invariant sets greatly simplifies the analysis of the dynamics. The principal tool available is the Stable Manifold Theorem [14], which states that for a hyperbolic invariant set, the distributions $E^{s}$ and $E^{u}$ are in fact tangent to global stable and unstable manifolds: manifolds, all of whose points have the same backwards and forwards (resp.) asymptotic behavior. See any of the standard texts (e.g., [12]) for further information and examples.

\section{Fixed points}

In analyzing the dynamics and topology of a flow, one examines dynamical $n$ skeleta of increasing dimension: first the fixed points, then periodic and connecting orbits, lastly higher-dimensional invariant manifolds and attractors. This section concerns the typical distribution of fixed points for plane field flows.

Lemma 2.1. Given $\eta$ a $C^{r}$ plane field on $M^{3}$ and $p \in M$ there exists a neighborhood $U \cong \mathbb{R}^{3}$ of $p$ along with local coordinates $(x, y, z)$ on $U$ such that $\eta=\operatorname{ker}(\alpha)$, where $\alpha$ is a one-form given by

$$
\alpha=d z+g(x, y, z) d y
$$

for some function $g$ which vanishes at the origin. The space $\Gamma^{r}\left(\left.\eta\right|_{U}\right)$ of $C^{r}$ sections of $\eta$ on $U$ is isomorphic to $C^{r}\left(\mathbb{R}, C^{r}\left(\mathbb{R}^{2}, \mathbb{R}^{2}\right)\right)$, the space of $C^{r}$ arcs of $C^{r}$ planar vector fields.

Proof. That $\alpha$ exists is easy to derive (and is stated in [7]): choose coordinates $(x, y, z)$ so that $\partial / \partial z$ is transverse to $\eta$ on $U$. Then, after rescaling, $\eta$ is the kernel of $d z+f(x, y, z) d x+g(x, y, z) d y$. By a change of variables, one can eliminate $f$ and remove constant terms in $g$.

Parameterize $U$ as $\left\{\mathbb{R}^{2} \times\{z\}: z \in \mathbb{R}\right\}$. Given any 1-parameter family of functions $F_{z}: \mathbb{R}^{2} \rightarrow \mathbb{R}^{2}$, there is a well-defined vector field on $U$ given by

$$
\begin{aligned}
& \dot{x}=f_{1}(x, y, z) \\
& \dot{y}=f_{2}(x, y, z) \quad \text { where } F_{z}(x, y)=\left(f_{1}(x, y, z), f_{2}(x, y, z)\right) \text {, } \\
& \dot{z}=-g(x, y, z) f_{2}(x, y, z)
\end{aligned}
$$

which lies within $\eta$ by Equation 2. Similarly, any vector field on $U$ contained in $\eta$ induces a 1-parameter family of planar vector fields $F_{z}: \mathbb{R}^{2} \rightarrow \mathbb{R}^{2}$ by inverting 
the above procedure. Since $\partial / \partial z$ is always transverse to $\eta$, zeros of $F_{z}$ correspond precisely with zeros of the induced vector field in $\eta$. Note finally that the correspondence is natural with respect to the $C^{r}$-topology (nearby families of planar vector fields induce nearby plane field flows and vice versa).

Proposition 2.2. Let $\eta$ be a $C^{r}(r \geq 1)$ plane distribution on $M$ a compact 3-manifold, and let $\Gamma(\eta)$ denote the space of $C^{r}$ sections of $\eta$. Then on an open dense subset of $\Gamma(\eta)$, the fixed point set is a smooth finite link of embedded circles.

Proof. From the Transversality Theorem (see [13, p. 74]) we know there is an open dense subset of sections of $\eta$ which are transverse to the zero section. The proposition clearly follows.

Corollary 2.3. Let $X$ be any vector field on $M^{3}$ contained in the distribution $\eta$. Then any fixed point of $X$ is nonhyperbolic.

Proof. Hyperbolic fixed points are isolated and persist in $C^{1}$-neighborhoods of vector fields; hence, they cannot be perturbed to yield circles of fixed points.

To analyze the dynamics near a curve of singularities, we show that for all but finitely many points, the dynamics are transversally hyperbolic; i.e., after ignoring the nonhyperbolic direction along the curve, the flow is hyperbolic along the tangent plane transverse to the curve. We then turn to classify the (codimension-1) bifurcations in the transverse behavior along a curve of singularities.

Proposition 2.4. Let $X \subset \eta$ be a $C^{r}(r \geq 2)$ section of a $C^{r}$ plane field $\eta$. Then on a residual set of such vector fields, Fix $(X)$ is a link $L$ which is transversally hyperbolic with respect to all but finitely many $p \in L$.

Proof. By a standard argument (see [13, p. 74]) is suffices to show that there is an open cover $\left\{U_{i}\right\}$ of $M$ for which there is a residual set of sections of $\left.\eta\right|_{U_{i}}$ with the desired property. Cover each $p \in M$ by a chart as in Lemma 2.1. On each chart, consider the map from $\mathbb{R}^{3} \rightarrow \mathbb{R}^{2}$ induced by a section of $\eta$. Extend this to a map into the 1-jet space $J^{1}\left(\mathbb{R}^{3}, \mathbb{R}^{2}\right)$ to capture information about the linearization of the flow. One may easily find a codimension three stratified subset $S$ of $J^{1}\left(\mathbb{R}^{3}, \mathbb{R}^{2}\right)$ on which a section will both vanish and be transversally nonhyperbolic. Thus by the Jet Transversality Theorem for $C^{2}$ maps we obtain a residual subset of sections of $\eta$ whose 1-jets transversally intersect $S$ at isolated points (which clearly must lie on $L$ ).

Corollary 2.5. Under the hypotheses of Proposition 2.4, the singular link $L$ is transverse to $\eta$ at all but a finite number of points. 
Proof. If the curve of singularities $S$ is tangent to the plane field $\eta$ at a point $p$, then $p$ is not transversally hyperbolic since the eigenvalue whose eigenvector points in the direction transverse to $\eta$ is zero (the vector field can have no component in the direction transverse to $\eta$ ).

It is now a simple matter to classify the points at which the vector field is not transversally hyperbolic to the equilibria. Thanks to Lemma 2.1, this analysis reduces simply to bifurcation theory of fixed points in planar vector fields. In particular, there are precisely two ways in which a (generic) $X \subset \eta$ can fail to be transversally hyperbolic at a point.

Given any singular point $p \in S$, the transverse dynamics is characterized by the pair of transverse eigenvalues for the linearized flow: $\lambda^{x}$ and $\lambda^{y}$. Transverse hyperbolicity fails if and only if one or both of these eigenvalues has zero real part. Generically, this can occur in two distinct ways. First, $\lambda^{x}$ and $\lambda^{y}$ may be both real, and one of them goes transversally through zero: this is a saddle-node bifurcation. Second, $\lambda^{x}$ and $\lambda^{y}$ may be a complex conjugate pair of eigenvalues which together pass through the imaginary axis transversally: this is a Hopf bifurcation. Again, these names correspond with analogous bifurcations of fixed points in planar vector fields.

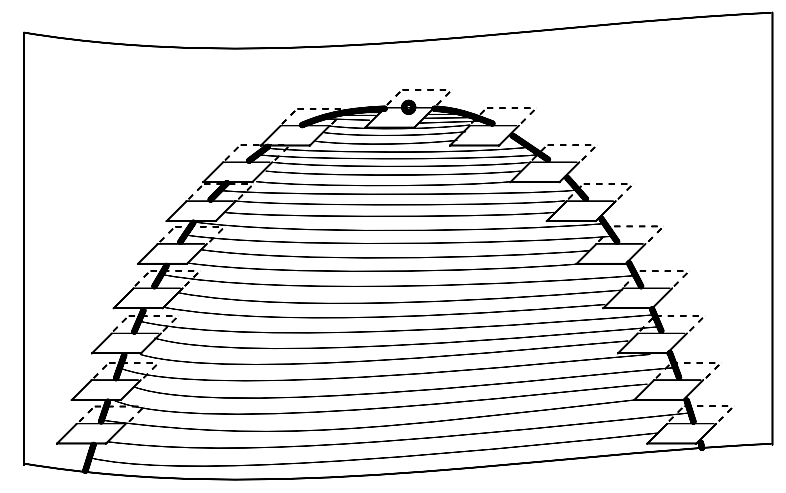

Figure 1.

A saddle-node bifurcation of singularities in a plane field flow.

Proposition 2.6. In the unfolding of a $C^{r}$-generic $(r \geq 2)$ saddle node bifurcation on a curve of fixed points in a plane field flow, there is a quadratic tangency between the plane field and the fixed point curve, along with a one-parameter family of heteroclinic connections between fixed points limiting onto the bifurcation point, as in Figure 1.

Proof. As per Lemma 2.1, choose a coordinate system $(x, y, z)$ on a neighborhood of the bifurcation point $p$ so that $\partial / \partial z$ is everywhere transverse to the plane field 
$\eta$. It is also clearly possible (via the Stable Manifold Theorem) to choose coordinates so that the $x$-direction corresponds to the eigenvector for the transversally hyperbolic eigenvalue $\lambda^{x}$.

By Lemma 2.1, the unfolding of this codimension-1 fixed point in a plane field flow corresponds to the codimension- 1 unfolding of a generic fixed point in a planar vector field having one hyperbolic eigenvalue and one eigenvalue with zero real part. The unfolding of the planar saddle-node is conjugate to the system [12]

$$
F_{z}: \begin{aligned}
& \dot{x}=\lambda^{x} x \\
& \dot{y}=z-a y^{2}
\end{aligned}
$$

for some $a \neq 0$, which, under Equation 3, corresponds to the vector field within $\eta$

$$
\begin{aligned}
& \dot{x}=\lambda^{x} x \\
& \dot{y}=z-a y^{2} \\
& \dot{z}=-g(x, y, z)\left(z-a y^{2}\right)
\end{aligned}
$$

The curve of fixed points is thus a parabola tangent to $\eta$ at the bifurcation point.

To show the existence of a family of heteroclinic curves from one branch of the parabola to the next, note that the planar vector fields $F_{z}$ have precisely this 1-parameter family of orbits. Upon "suspending" to obtain a vector field within $\eta$, the orbits remain, since the expression for $\dot{z}=-g(x, y, z)\left(z-a y^{2}\right)$ vanishes at $(0,0,0)$; hence, $\dot{z}$ is bounded near zero in a neighborhood of the bifurcation value and the integral curves within the invariant plane $\dot{x}=0$ must connect.

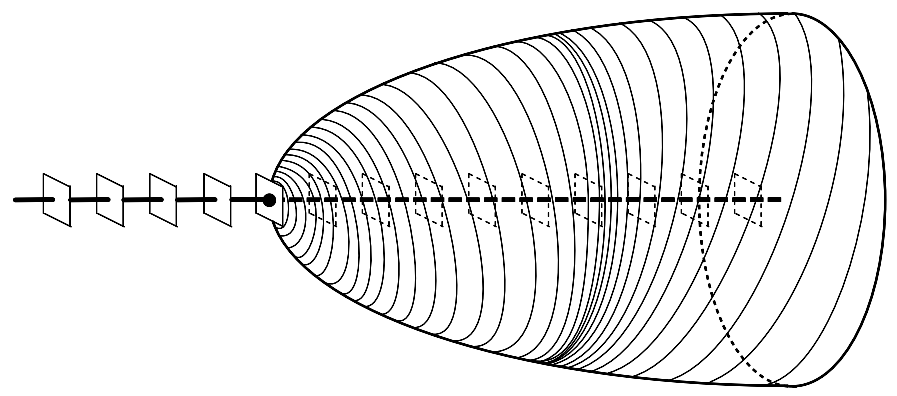

Figure 2 .

A Hopf bifurcation of singularities in a plane field flow.

Proposition 2.7. In the unfolding of a $C^{r}$-generic $(r \geq 4)$ codimension-one Hopf bifurcation on a curve of fixed points in a plane field flow, there is an invariant attracting or repelling paraboloid which opens along the curve of fixed points as in Figure 2. 
Proof. Since only the real portion of the transverse eigenvalues vanish, the curve of fixed points is transverse to the plane field in a neighborhood of the bifurcation point $p$. Hence, choose coordinates as per Lemma 2.1 such that the curve of fixed points is the $z$-axis and the bifurcation point is at $(0,0,0)$. Again, by Lemma 2.1, this bifurcation in a plane field flow corresponds precisely to the codimension-one Hopf bifurcation of planar vector fields, conjugate to the truncated normal form [12]

$$
F_{z}: \begin{aligned}
& \dot{r}=z r+a r^{3} \\
& \dot{\theta}=\omega
\end{aligned}
$$

where we have transformed $(x, y)$ to polar coordinates and the constant $a$ is (in the codimension- 1 scenario) nonzero. Solving this equation for $\dot{r}=0$ yields the paraboloid $r=\sqrt{-z / a}$, which is either attracting or repelling, depending on the sign of the coefficient $a$. By translating Lemma 2.1 into polar coordinates, it follows that $\dot{z}$ is of order $r^{2}$, which is less than $\dot{r}$; hence, adding the dynamics in the $z$-component affects neither the existence nor the attracting/repelling nature of the invariant paraboloid; however, unlike the planar case, the paraboloid is not necessarily fibered with closed curves. In general, orbits will spiral about the paraboloid.

Remark 2.8. We note that saddle-node or Hopf bifurcations must occur in pairs, since the fixed point curves are circles and the index at a bifurcation changes. However, in the case where there are no saddle-node or Hopf bifurcations along the singular curve, the flow is everywhere transversally hyperbolic, and the index of the fixed points (source, saddle, or sink) is constant along the curve.

We conclude with the definition of a nondegenerate vector field tangent to a plane field, and prove that such vector fields are generic.

Definition 2.9. A nondegenerate section of a plane field $\eta$ is a vector field $X \subset \eta$ whose fixed point set is a link having transversally hyperbolic dynamics at all but a finite number of points, at which the degeneracies are codimension one.

Proposition 2.10. Nondegenerate fields are generic (residual in the $C^{r}$ topology $r \geq 4$ ) within the space of sections to a $C^{r}$ plane field $\eta$.

Proof. We simply repeat the argument in the proof of Proposition 2.4 using Propositions 2.6 and 2.7 .

\section{Round handles and gradients}

Let $X$ be a nondegenerate vector field contained in the plane field $\eta$. The goal of the remaining sections is to understand restrictions on the topology of 3-manifolds 
supporting plane field flows which are forced by prescribed dynamics. A wellknown example of this occurs in the case of Anosov flows: certain three-manifolds are prohibited from carrying Anosov dynamics. In contrast, we examine obstructions associated to the simplest kinds of dynamics: gradient plane field flows. We show that only certain topologically "simple" manifolds support such dynamics. This will lead us to further knot-theoretic obstructions based on the singular links in a plane field flow. An old theme is played out: when the dynamics of $X$ are simple, the links associated to it are simple.

Lemma 3.1. Let $M$ denote an oriented Riemannian 3-manifold and $X=-\nabla \Psi$ $a C^{r}(r \geq 2)$ gradient vector field which lies within a $C^{r}$ plane field $\eta$ on $M$. Then $\Psi$ is constant on each connected component of Fix $(X)$, the fixed point set of $X$. Furthermore, if $c$ is a regular value of $\Psi$, then $\Psi^{-1}(c)$ is a disjoint union of tori transverse to both $X$ and $\eta$.

Proof. Each component of $\operatorname{Fix}(X)$ is a compact connected set of critical points for $\Psi$, whose image under $\Psi$ is a compact connected subset of $\mathbb{R}$ having measure zero, by the Morse-Sard Theorem. For $c$ regular, $\Psi^{-1}(c)$ is a disjoint union of smooth surfaces, and $X$ is transverse to each component since $X$ is a gradient field. Hence, the plane field $\eta$ is everywhere transverse to $\Psi^{-1}(c)$ and the resulting line field given by the intersection of $\eta$ and the tangent planes to $\Psi^{-1}(c)$ in $\left.T M\right|_{\Psi^{-1}(c)}$ is nonsingular. Thus, the Euler characteristic of each component of $\Psi^{-1}(c)$ is zero. The transverse vector field $X$ gives an orientation to the surface, which excludes from consideration the Klein bottle.

Grayson and Pugh [11] give examples of $C^{\infty}$ functions on $\mathbb{R}^{3}$ whose critical points consist of a smooth link, yet for which the level sets are usually not tori: see Remark 4.5.

The above mentioned restrictions on gradient plane fields translate into very precise conditions on the topology of the underlying three-manifold. The fact that the manifold consists of a finite number of thick tori $T^{2} \times[0,1]$ glued together in ways prescribed by $\Psi$ implies that the manifold can be decomposed into solid tori in a canonical fashion: this phenomenon was identified and analyzed by Asimov and Morgan in the 1970's [1, 18] in a completely different context.

Definition 3.2. A round handle (or $\mathrm{RH}$ ) in dimension three is a solid torus $H=D^{2} \times S^{1}$ with a specified index and exit set $E \subset T^{2}=\partial\left(D^{2} \times S^{1}\right)$ as follows: index 0: $E=\emptyset$.

index 1: $E$ is either (1) a pair of disjoint annuli on the boundary torus, each of which wraps once longitudinally; or (2) a single annulus which wraps twice longitudinally.

index 2: $E=T^{2}$. 
Definition 3.3. A round handle decomposition (or RHD) for a manifold $M$ is a finite sequence of submanifolds

$$
\emptyset=M_{0} \subset M_{1} \subset \cdots M_{n}=M
$$

where $M_{i+1}$ is formed by adjoining a round handle to $\partial M_{i}$ along the exit set $E_{i+1}$ of the round handle. The handles are added in order of increasing index.

Asimov and Morgan $[1,18]$ used round handles to classify nonsingular MorseSmale vector fields: that is, vector fields whose recurrent sets consist entirely of a finite number of hyperbolic closed orbits with transversally intersecting invariant manifolds.

Theorem 3.4. Let $\eta$ denote a $C^{r}(r \geq 2)$ plane field on $M^{3}$ (compact) with $X \subset \eta$ $a C^{r}$ nondegenerate gradient vector field. Then the set of fixed points for $X$ forms the cores of a round handle decomposition for $M$. Furthermore, the indices of the fixed points correspond to the indices of the round handles, and $X$ is transverse to $\partial M_{i}$ for all $i$.

Proof. Let $L$ denote the set of fixed points for $X=-\nabla \Psi$ : this is an embedded link. We first show that every fixed point is transversally hyperbolic. From Remark 2.8, the only non-transversally hyperbolic points must occur as Hopf bifurcations or saddle-node bifurcations. Hopf bifurcations are associated to complex transverse eigenvalues, which cannot exist in a gradient flow. Similarly, a saddle-node bifurcation introduces a one-parameter family of heteroclinic connections as in Figure 1. This also cannot occur in a gradient flow, since by Lemma 3.1 we have the function $\Psi$ constant on the curve of fixed points. The orbits of the flow which necessarily connect one side to the other cannot be obtained by flowing down a gradient. Hence, each singular curve is transversally hyperbolic with constant index.

Choose $N$ a small tubular neighborhood of $L$ in $M$ and let $f$ denote a bump function in $N$ which evaluates to 1 on $L$ and is zero outside of $N$. Orient the link $L$ and perturb $X$ to the new vector field $X+\epsilon f \frac{\partial}{\partial z}$, where $\frac{\partial}{\partial z}$ denotes the unit tangent vector along $L$. This yields a nonsingular flow which has $L$ as a set of hyperbolic closed orbits and no other recurrence. After a slight perturbation to remove any nontransverse intersections of stable and unstable manifolds to $L$, this vector field is a nonsingular Morse-Smale field with periodic orbit link $L$. The work of Morgan [18] then implies that $L$ forms the cores of a round handle decomposition for $M$, where the index of each handle corresponds to the transverse index of the curve of fixed points (source, saddle, or sink). In [18] it is moreover shown that the nonsingular Morse-Smale vector field is transverse to each $\partial M_{i}$; since the neighborhood $N$ is very small, this transversality remains in effect for $X$.

Corollary 3.5. Gradient flows on plane fields in three-manifolds lie on the boundary of the space of nonsingular Morse-Smale fields. 
Proof. In the proof of Theorem 3.4, let $\epsilon \rightarrow 0$. This gives a one-parameter family of nonsingular Morse-Smale flows which converges to the gradient plane-field flow.

Corollary 3.6. Non-gradient dynamics is a generic condition in the space of plane field flows on an irreducible non-graph three-manifold (e.g., a hyperbolic 3manifold).

Proof. By the work of Morgan [18], round handle decompositions of irreducible three-manifolds exist only for the class of graph-manifolds.

Recall that a graph manifold is a three-manifold given by gluing together Seifert-fibered spaces along essential torus boundaries. Examples include $S^{3}$, lens spaces, and manifolds with many $S^{2} \times S^{1}$ connected summands. The property of being composed of Seifert-fibered pieces (i.e., a graph manifold) is relatively rare among three-manifolds, the "typical" irreducible three-manifold being composed of hyperbolic pieces.

Remark 3.7. We may push Theorem 3.4 a bit further. Let $\phi^{t}$ be a plane field flow whose chain-recurrent set consists entirely of transversally hyperbolic curves of fixed points and a finite set of hyperbolic periodic orbits (note that hyperbolic periodic orbits can easily live within plane fields, even within nowhere integrable plane fields). This situation is, after the class of gradient flows, the next simplest scenario dynamically. Then, by the same proof, the connected components of the entire chain-recurrent set must form the cores of a round-handle decomposition. Hence, the additional dynamics forced upon plane field flows in a non-graph manifold is something other than hyperbolic periodic orbits.

\section{The link of singularities}

We have shown that fixed points of plane field flows appear in links. The natural question is which links can arise as the singular points, and what dependence is there upon the dynamics of the plane field flow. For nondegenerate gradient fields, it is an immediate corollary of Theorem 3.4 that the singular link is a collection of fibers in the Seifert-fibered portions of a graph manifold. We can be more specific, however, in the special case of $S^{3}$. We recall two standard operations for transforming simple knots into more complex knots: see Figure 3 for an illustration.

Definition 4.1. Let $K$ be a knot in $S^{3}$. Then the knot $K^{\prime}$ is said to be a $(p, q)$ cable of $K$ if $K^{\prime}$ lives on the boundary of a tubular neighborhood of $K$, wrapping about the longitude (along $K$ ) $p$-times and about the meridian (around $K$ ) $q$ - 
times. Let $K$ and $J$ be a pair of knots in $S^{3}$. Then the connected sum, denoted $K \# J$, is defined to be the knot obtained by removing from each a small arc and identifying the endpoints along a band as in Figure 3.
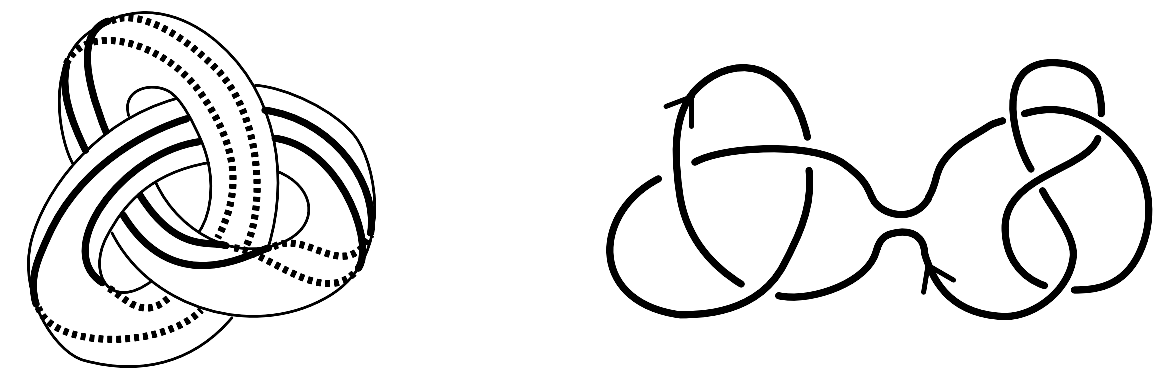

Figure 3.

Operations to generate zero-entropy knots: (left) cabling; (right) connected sum.

Definition 4.2. The zero-entropy knots are the collection of knots generated from the unknot by the operations of cabling and connected sum; i.e., it is the minimal class of knots closed under these operations and containing the unknot.

Zero-entropy knots are relatively rare among all knots: e.g., none of the hyperbolic knots (such as the figure-eight knot, whose complement has a hyperbolic structure) are zero-entropy. The title stems from the often-discovered fact (see [8] for history) that such knots are associated to three-dimensional flows with topological entropy zero.

Corollary 4.3. Given a nondegenerate gradient plane field flow on $S^{3}$, every component of the fixed point link is a zero-entropy knot.

Proof. Wada [22] classifies the knot types for cores of all round handle decompositions on $S^{3}$. Each component is a zero-entropy knot.

Remark 4.4. Much more can be said: Wada in fact classifies all possible links which arise as round handle cores on all graph manifolds. This class of zeroentropy links is an extremely restricted class, which lends credence to the motto that simple dynamics implicate simple links in dimension three. We note this same class of links appears independently in the study of nonsingular Morse-Smale flows, suspensions of zero-entropy disc maps, and in Bott-integrable Hamiltonian flows with two degrees of freedom.

Remark 4.5. It is possible to construct gradient flows on $S^{3}$ (for example) in which the fixed point set is an embedded link which is not a zero-entropy link. Let 
$L_{1}$ and $L_{2}$ denote any pair of links in $S^{3}$ which each have at least three components. Grayson and Pugh [11] prove the existence of $C^{\infty}$ functions $\Psi_{1}, \Psi_{2}: \mathbb{R}^{3} \rightarrow \mathbb{R}$ which have $L_{1}$ and $L_{2}$ as the (respective) sets of critical points. Moreover, these functions are proper and, for large enough $c \in \mathbb{R}$, the inverse image of $c$ is a smooth 2-sphere near infinity. Hence, we may consider the balls $B_{i}$ bounded by $\Psi_{i}^{-1}(c)$ and glue them together along the boundaries, obtaining $S^{3}$. The resulting function $\Psi$ given by $\Psi_{1}$ on $B_{1}$ and $-\Psi_{2}+2 c$ on $B_{2}$ has as its gradient flow the split (unlinked) sum of $L_{1}$ and $L_{2}$ as its fixed points. Thus, this flow cannot live within a plane field.

It is not ostensibly clear that every zero-entropy link in $S^{3}$ is realized as the zero set of a gradient flow within a plane field. We close this section with a realization theorem for such flows which shows that, in fact, a particular subclass of round-handle decompositions (and, hence, zero-entropy links) is realized.

Lemma 4.6. If $X$ is a nondegenerate gradient field on $M$ contained in the plane field $\eta$, then each index-1 round-handle $H$ in the decomposition must be attached to $\partial M_{i}$ along annuli which are essential (homotopically nontrivial) in $\partial M_{i}$.

Proof. Assume that $M_{i}$ is the $i$ th stage in a round handle decomposition, and that $H$ is an index-1 round handle with an exit annulus $E$ which is essential in $\partial H$ by definition. By Theorem 3.4, the intersection of $\eta$ with $\partial M_{i}$ is always transverse. Thus, if $H$ is attached to $M_{i}$ along an annulus $A \subset \partial M_{i}$, then the foliations given by the intersections of $\eta$ with the tangent planes to $A$ and $E$ respectively must match under the attachment. We claim this is impossible when $A$ is homotopically trivial in $\partial M_{i}$.

Define the index of a smooth (oriented) curve $\gamma$ in an orientable surface with a (nonsingular, oriented) foliation $\mathcal{F}$ to be the degree of the map which associates to each point $p \in \gamma$ the angle between the tangent vectors to $\gamma$ and $\mathcal{F}$ at $p$. This index is independent of the metric chosen and also invariant under homotopy of $\gamma$ or of $\mathcal{F}$; hence, we can speak of the index of an annulus in a surface with foliation.

When $A$ is homotopically trivial, the index must be equal to \pm 1 , since a foliation is locally a product. However, the index of the exit annulus $E \subset \partial H$ must be zero as follows. Under the gradient field $X$, the core of the 1-handle is a curve $\kappa$ of fixed points with transverse index 1 whose unstable manifold $W^{u}(\kappa)$ intersects $\partial H$ transversally along the core of the exit set $E$. Deformation retract $E$ to a small neighborhood of $\mathcal{W}^{u}(\kappa) \cap \partial H$ - here, the intersections with $\eta$ are always transverse. Next, homotope the annulus to a neighborhood of $\kappa$ by integrating the gradient field $X$ backwards in time. This has the effect of taking the annulus transverse to $W^{u}(\kappa)$ and sliding along $W^{u}(\kappa)$ back to $\kappa$. Since $X$ points outwards along $W^{u}(\kappa)$, the image of the annulus $E$ under the homotopy is always transverse to $X$, and hence to $\eta$. The fact that $\eta \pitchfork \kappa$ then implies that the foliation on $E$ induced by $\eta$ must be homotopic through nonsingular foliations to a product foliation by intervals on the annulus, which implies that the longitudinal annulus 
$E$ has index zero. Note that this works for exit sets $E$ which wrap any number of times about the longitude of $H$ (to cover both types of index-1 round handles).

Hence, any round-handle decomposition which is realizable as a gradient plane field flow must have all 1-handles attached along essential annuli. We call such a round-handle decomposition essential.

Theorem 4.7. Let $M$ be a compact 3-manifold with $L$ an indexed link. Then $L$ is realized as the indexed set of zeros for some nondegenerate gradient plane field flow on $M$ if and only if $L$ is the indexed set of cores for an essential RHD on $M$.

Proof. The necessity is the content of Lemma 4.6. Given any essential RHD, we construct a corresponding plane field gradient flow. One may begin with the fact proved by Fomenko that any essential round-handle decomposition can be generated by a vector field $X$ integrable via a Bott-Morse function $\Psi: M \rightarrow \mathbb{R}$ with all critical sets being circles (see [3] for a detailed exposition). After choosing a metric on $M$ we claim that $-\nabla \Psi$ lives within the plane field $\eta$ orthogonal to $X$. Indeed, away from $L$ the plane field $\eta$ will be spanned by $\nabla \Psi$ and $\nabla \Psi \times X$ since these are linear independent vectors orthogonal to $X$ (recall $X$ is tangent to the level sets of $\Psi$ ). Thus $-\nabla \Psi$ clearly lies in $\eta$ on the complement of $L$. Along $L$ the gradient $-\nabla \Psi$ lies in $\eta$ since it is zero.

\section{Flows on foliations and contact structures}

\subsection{Foliations}

In the case where our given plane field has some geometrical property, we may further restrict the types of round-handle decompositions which may contain a gradient flow. For example, if the plane field $\eta$ is integrable, it determines a foliation on the manifold. In this subsection, we note that, in this case, $S^{3}$ cannot support such a gradient flow. This result, which is an obvious corollary of Novikov's Theorem on foliations, generalizes to other three-manifolds.

Recall from the theory of foliations on three-manifolds (see, e.g., [9]) that a Reeb component is a foliation of the solid torus $D^{2} \times S^{1}$ that consists of the boundary $T^{2}$ leaf along with a one-parameter family of leaves, each homeomorphic to $\mathbb{R}^{2}$ and limiting onto the boundary with nontrivial holonomy, as in Figure 4. A codimension-one foliation of a three-manifold is taut if there do not exist Reeb components or "generalized" Reeb components (see, e.g., [7] for definitions). An equivalent definition of taut is that given any leaf $\mathcal{L}$ there exists a closed curve through $\mathcal{L}$ transverse to the foliation.

It is straightforward to show that gradient fields must lie within taut foliations: 


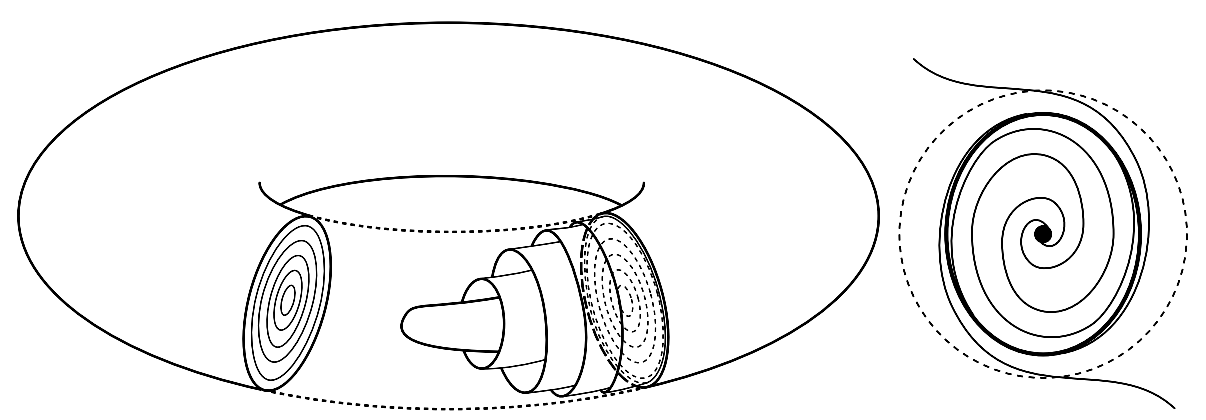

Figure 4.

A Reeb component in a foliation on a 3-manifold (left) can be perturbed into an overtwisted contact structure (right).

Theorem 5.1. Let $\mathcal{F}$ denote a codimension-1 foliation on a compact threemanifold $M$ which contains a nondegenerate gradient vector field $X$. Then $\mathcal{F}$ is taut.

Proof. Assume that $X=-\nabla \Psi$ is a nondegenerate gradient field on a foliation $\mathcal{F}$. For $\mathcal{L}$ a leaf of $\mathcal{F}$, the restriction of $X$ to $\mathcal{L}$ must also be a gradient flow. In the case where $\mathcal{L}$ is compact, there must be a nondegenerate fixed point of $X$ on $\mathcal{L}$ which lies on a circle of fixed points transverse to $\mathcal{F}$ (note that in the case of a boundary torus in a Reeb component, this is an immediate contradiction). In the case where $\mathcal{L}$ is not a compact leaf, choose some nontrivial path $\gamma \subset \mathcal{L}$ whose endpoints are directly above one another in a local product chart. Then, by perturbing $\gamma$ to be transverse to $\mathcal{F}$, we may close it up to a transverse loop through $\mathcal{L}$.

This result can be greatly improved by considering the holonomy of the foliation. Recall that the holonomy of any closed curve $\gamma: S^{1} \rightarrow \mathcal{L}$ in a leaf $\mathcal{L}$ of a codimension-one foliation $\mathcal{F}$ is the germ of the Poincaré map associated to the characteristic foliation on an annulus transverse to $\mathcal{L}$ along $\gamma$. The holonomy of a curve is an invariant of its homotopy class within the leaf. A foliation has vanishing holonomy if the holonomy of every curve $\gamma$ is trivial (the identity).

Theorem 5.2. Any closed orientable three-manifold $M$ containing a nondegenerate gradient field within a $\left(C^{r}\right.$ for $\left.r \geq 2\right)$ codimension-one foliation is a surface bundle over $S^{1}$.

Proof. Suppose that $M$ admits a foliation $\mathcal{F}$ which supports a nondegenerate gradient field. Then, by Theorem 3.4, $M$ has a round handle decomposition where all the regular tori are transverse to the foliation. The foliation on each round handle is equivalent to the product foliation by discs on $D^{2} \times S^{1}$, since these solid tori are filled with leaves transverse to the boundary each having a gradient 
flow with a single fixed point. We show in subsequent steps that the foliation $\mathcal{F}$ may be modified within the round handle structure so that the new foliation $\mathcal{F}^{\prime}$ has no holonomy. Once we show this, the celebrated theorem of Sacksteder implies that this foliation must be topologically conjugate to the kernel of a closed nondegenerate 1-form on $M$ [20]. The existence of this 1-form implies, via the theorem of Tischler [21], that $M$ must be a surface bundle over $S^{1}$. We illustrate in Figure 5 below that it is possible to have gradient fields within a foliation having holonomy, so it is truly necessary to develop the following modification procedure, which makes use of "shearing" the foliation along 1-handles (cf. [4]).

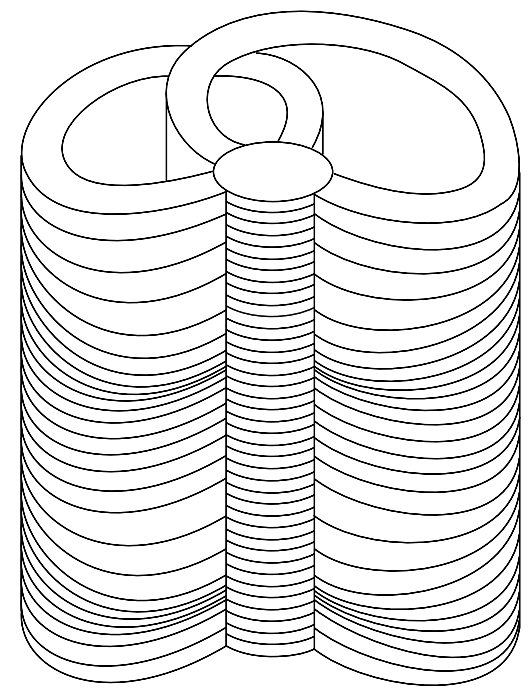

Figure 5.

A foliated RHD on $T^{2} \times S^{1}$ with holonomy along each 1-handle: the 2-handle has been removed and the $S^{1}$-factor cut open, revealing a pair of 1 -handles attached to a 0 -handle with inverse attaching maps.

Denote by $M_{0}$ the (disjoint) union of all the 0 -handles and by $M_{i}(1 \leq i \leq N)$ the subsequent stages in the decomposition:

$$
M_{i}=\left(M_{i-1} \sqcup H_{i}\right) / \phi_{i},
$$

where $H_{i}$ is the $i$ th 1 -handle and $\phi_{i}: E_{i} \hookrightarrow \partial M_{i-1}$ is the attaching map on the exit set $E_{i} \subset H_{i}$. Recall that each exit set $E_{i}$ is either one or two annuli and that the boundary of each $M_{i}, \partial M_{i}$, is the disjoint union of a collection of tori.

For each 1-handle $H_{i}$, let $W_{i}^{u}$ denote the (2-dimensional) unstable manifold to the core of $H_{i}$. Modify the round handle structure so that each $H_{i}$ is very "thin" - that is, each $H_{i}$ is restricted to a small neighborhood of $W_{i}^{u}$, appending the 
"leftover" portion to the neighboring 2-handles. Denote by

$$
\mathrm{Bd}_{i}=\partial M_{0} \bigcup_{j=1}^{i} W_{j}^{u}
$$

the 2-complex given by the union of all the 0-handle boundaries and unstable manifolds of the 1-handles in $M_{i}$.

Claim 1. $\mathcal{F}$ has vanishing holonomy if the restriction of $\mathcal{F}$ to $\mathrm{Bd}_{N}$ has vanishing holonomy.

Proof 1. Let $\gamma$ denote a loop within a leaf $\mathcal{L}$ of $\mathcal{F}$. Then the restriction of $\mathcal{L}$ to each $k$-handle is a collection of disjoint discs whose boundaries lie in the union of 0 - and 1-handles. Push $\gamma$ to these boundaries and, since $\partial H_{i}$ is very close to $W_{i}^{u}$, perturb $\gamma$ to lie within $W_{i}^{u}$ for each $H_{i}$ it intersects. Since $\mathrm{Bd}_{N}$ is transverse to $\mathcal{F}$, we may choose the transverse annulus $A$ containing $\gamma$ to lie within this set. $\square$

In what follows, we consider holonomy on the 2-complex $\mathrm{Bd}_{N}$, keeping in mind that the 1-handles are actually thin neighborhoods of the 2-cells $W_{i}^{u}$. The holonomy on each component of $\partial M_{i}$ is equivalent to that on the corresponding piece of $\mathrm{Bd}_{N}$ since each $H_{i}$ has a product foliation.

Claim 2. The maps $\left\{\phi_{i}\right\}_{1}^{N}$ may be isotoped so that the induced foliation $\mathcal{F}^{\prime}$ on $\mathrm{Bd}_{N}$ is without holonomy.

Proof 2. It suffices to show that the foliation restricted to each $\partial M_{i}$ is without holonomy (a product foliation): we proceed by induction on $i$. On the boundary of $M_{0}$ the foliation $\mathcal{F}$ restricts to a product foliation by circles. Assume as an induction hypothesis a lack of holonomy on $\partial M_{i-1}$. There are three cases to consider: (1) $H_{i}$ is an orientable handle with attaching circles in the same component of $\partial M_{i-1} ;(2) H_{i}$ is orientable with attaching circles in two distinct components of $\partial M_{i-1}$; and $(3) H_{i}$ is nonorientable.

Case (1): Let $C_{ \pm}$denote the circles in the selected component $T$ of $\partial M_{i-1}$ along which $W_{i}^{u}$ is attached. Note $C_{ \pm}$divides $T$ into two annuli $A_{0}$ and $A_{1}$. After fixing a diffeomorphism from $C_{+}$to $C_{-}$there is a "handle holonomy map" $f_{H}: C_{+} \rightarrow C_{-}$ which is the diffeomorphism given by sliding along leaves on $\phi_{i}\left(H_{i}\right)$. There are corresponding "boundary holonomy maps" $f_{j}: C_{+} \rightarrow C_{-}$given by sliding along leaves on $A_{j}$. Isotope $\phi_{i}$ on $C_{+}$so that $f_{H}$ equals $f_{0}$ up to a rigid rotation (which is necessary in order to add subsequent handles along curves transverse to $\mathcal{F}$ see Claim 3). The holonomy on the two new components of $\partial M_{i}$ is determined by taking the transverse curve $C_{+}$(actually one must take a parallel copy of $C_{+}$that sits in $\left.\partial M_{i}\right)$ as a section. These holonomy maps factor as $f_{1}^{-1} \circ f_{H}$ and $f_{H}^{-1} \circ f_{0}$; however, the holonomy along $C_{+}$within $\partial M_{i-1}$ is a map of the form $f_{1}^{-1} \circ f_{0}$, 
which, by induction, is a rigid rotation. Hence, up to rotations, $f_{0}=f_{1}$. Since we chose $f_{H}=f_{0}$ up to rotations the holonomy on $\partial M_{i}$ vanishes.

Case (2): If $H_{i}$ connects two disconnected boundary components of $M_{i-1}$, then the holonomy along $H_{i}$ will always cancel with itself as follows. Denote by $f_{H}: C_{+} \rightarrow C_{-}$the handle holonomy maps as before. Then the global holonomy map along $\partial M_{i}$ is of the form $g_{+} \circ f_{H} \circ g_{-} \circ f_{H}^{-1}$, where $g_{+}: C_{+} \rightarrow C_{+}$and $g_{-}: C_{-} \rightarrow C_{-}$are holonomy self-maps along loops in the two components of $\partial M_{i-1}$, and hence by induction, identity maps.

Case (3): If $H_{i}$ has connected exit set, the proof follows as in Case (1), since the handle must connect a single component of $\partial M_{i-1}$ to itself: isotope $\phi_{i}$ so that the handle holonomy map equals the holonomy map along the boundary up to a rigid rotation.

Claim 3. This "linearization" of $\mathcal{F}$ does not affect the topology of $M$.

Proof 3. Throughout the addition of the 1-handles, nothing about the topology of $M$ has changed, since the handle structure is identical - we modify only the foliation. However, after attaching the last 1-handle, the characteristic foliation on the boundary tori must be linear and rational, in order to glue in the 2-handles respecting the product foliation on their boundaries. The slopes of $\mathcal{F}$ restricted to $\partial M_{N}$ completely determine the topology of $M$ after adding the 2-handles (these are Dehn filling coefficients).

Hence, we must be able to linearize all of the attaching maps for the 1-handles without changing the boundary slopes at the end of the sequence. To do so, we preserve at every stage the rotation number of the holonomy maps $h_{i}$ which slide the attaching curves of $H_{i}$ along $\partial M_{i}$. Recall that to every diffeomorphism $f: S^{1} \rightarrow S^{1}$ is associated a rotation number $\rho_{f} \in \mathbb{R} / \mathbb{Z}$ which measures the average displacement of orbits of $f$ (see, e.g., [12]). When modifying $\phi_{i}$ to $\tilde{\phi}_{i}$ in the above procedure, we may compose $\tilde{\phi}_{i}$ with a rigid rotation by the angle necessary to preserve the rotation number of the holonomy map $h_{i}$ acting on the attaching curves in $\partial M_{i-1}$ (without adding further Dehn twists). This shearing maintains the average slope of the boundary foliation at each stage without adding holonomy. Hence, at the end of the 1-handle additions, when the original foliation had all boundary components with linear foliations of a particular fixed slope, the modified foliation also has linear boundary foliations with the same slope. Thus, adding the 2-handles is done using the same surgery coefficients, yielding the original manifold $M$ with a foliation having trivial holonomy.

Claims 1-3 complete the proof of Theorem 5.2.

Remark 5.3. Of course, not every surface bundle over $S^{1}$ may support a gradient field within a foliation: there is still the restriction that $M$ be a graph-manifold. This translates precisely into a condition on the monodromy map of the fibration - 
the monodromy must be of periodic (or reducibly periodic) type with respect to the Nielsen-Thurston classification of surface homeomorphisms. Any pseudo-Anosov piece in the monodromy forces hyperbolicity, contradicting the graph condition. It is not hard to see that any such bundle can be given a gradient field lying within each fiber $F$ of the bundle by choosing a Morse function $\phi: F \rightarrow \mathbb{R}$ which is equivariant with respect to the monodromy map.

Remark 5.4. All of the results of this section apply not only to gradient flows, but also to gradient-like flows, or flows for which there exists a function which decreases strictly along non-constant flowlines. The reason why nondegenerate gradient-like flows in foliations determine round-handle decompositions whereas for general plane fields they do not lies in the fact that the Hopf bifurcation of Proposition 2.7 cannot take place among gradient-like flows in the integrable case, while it can in the nonintegrable.

\subsection{Contact structures}

In contrast to the case of an integrable plane field, one may consider the class of contact structures, which has attracted interest in the fields of symplectic geometry and topology, knot theory, mechanics, and hydrodynamics.

Definition 5.5. A contact form on a three-manifold $M$ is a one-form $\alpha \in \Omega^{1}(M)$ such that the Frobenius integrability condition fails everywhere: that is,

$$
\alpha \wedge d \alpha \neq 0 \text {. }
$$

A contact structure on $M$ is a plane field $\xi$ which is the kernel of a locally defined contact form: that is,

$$
\xi_{p}=\left\{\mathbf{v} \in T_{p}: \alpha(\mathbf{v})=0\right\}
$$

for each $p \in M$.

Contact structures are thus maximally nonintegrable: the plane field is locally twisted everywhere. One may think of a contact structure as being an antifoliation, which leads one to suspect that the topology of the manifold may be connected to the geometry of the structure, as is often the case with foliations. Indeed, the contrast between foliations with Reeb components and those without Reeb components is reflected in the tight / overtwisted dichotomy in contact geometry (due primarily to Eliashberg [6] and Bennequin [2]).

Definition 5.6. Given a contact structure $\xi$ on $M$ and an embedded surface $F \subset M$, then the characteristic foliation $F_{\xi}$ is the (singular) foliation induced by the (singular) line field $\left\{T_{p} F \cap \xi_{p}: p \in F\right\}$. A contact structure $\xi$ is overtwisted if there exists an embedded disc $D \in M$ such that $D_{\xi}$ has a limit cycle, as in Figure 4 (right). A contact structure is tight if it is not overtwisted. 
The classification of contact structures follows along lines similar to that of codimension one foliations with or without Reeb components. An infinite number of homotopically distinct overtwisted contact structures exist on every closed orientable three-manifold $[17,16]$ and are algebraically classified up to homotopy [5]. Tight structures, on the other hand, are quite mysterious: e.g., it is unknown whether they exist on all three-manifolds.

Several examples of the similarity between tight contact structures and Reebless foliations are provided by the recent work of Eliashberg and Thurston [7]. For example, Reebless foliations can be perturbed into tight contact structures and foliations with Reeb components can be perturbed into overtwisted structures ( $c f$. Figure 4). Also, both Reebless foliations and tight structures satisfy a strong inequality restricting Euler classes. Tight structures are somewhat more general than Reebless foliations since the former can exist on $S^{3}[2]$ while the latter cannot [19]. Likewise, overtwisted structures are slightly more general than their foliation counterparts via the following observation, to be contrasted with Theorem 5.1:

Proposition 5.7. Any nondegenerate gradient field $X$ which lies within a tight contact structure $\xi$ on $M^{3}$ also lies within an overtwisted contact structure $\xi^{\prime}$ on $M^{3}$.

Proof. The canonical way to turn a tight structure into an overtwisted structure is by performing a Lutz twist $[16,17]$ on a simple closed curve $\gamma$ transverse to $\xi$. We execute a version of this twisting which respects a gradient field.

Given a gradient field $X \subset \xi$, choose a curve $\gamma$ of fixed points of index zero (sinks). Translate the function $\Psi$ whose gradient defines $X$ so that $\left.\Psi\right|_{\gamma} \equiv 0$. Since $\gamma$ is an index zero curve, $\Psi$ increases as one moves radially away from $\gamma$.

Let $N$ denote a tubular neighborhood of $\gamma$ whose boundary is a connected component of $\Psi^{-1}(\epsilon)$ for some $\epsilon>0$. Place upon $N$ the natural cylindrical coordinates $(\Psi, \theta, z)$. In analogy with Lemma 2.1, we may choose $\theta$ and $z$ so that $\left.\xi\right|_{N}$ is the kernel of the locally defined 1 -form

$$
\alpha=g(\Psi, \theta, z) d \theta+d z
$$

for some function $g$ with $g(0, \theta, z)=0$. The contact condition implies that

$$
\frac{\partial g}{\partial \Psi}>0
$$

Replacing this structure locally with the kernel of the form

$$
\alpha^{\prime}=\sin \left(\frac{\pi}{4}+\frac{2 \pi g}{g(\epsilon, \theta, z)}\right) g d \theta+\cos \left(\frac{\pi}{4}+\frac{2 \pi g}{g(\epsilon, \theta, z)}\right) d z
$$

yields a contact structure since

$\alpha^{\prime} \wedge d \alpha^{\prime}=\left[\cos \left(\frac{\pi}{4}+\frac{2 \pi g}{g(\epsilon, \theta, z)}\right) \sin \left(\frac{\pi}{4}+\frac{2 \pi g}{g(\epsilon, \theta, z)}\right)+\frac{2 \pi g}{g(\epsilon, \theta, z)}\right] \frac{\partial g}{\partial \Psi} d \Psi \wedge d \theta \wedge d z$, 
and this coefficient is positive by Equation 11. This contact structure agrees with that defined by $\alpha$ along the torus $\Psi=\epsilon$ since

$$
\left.\alpha^{\prime}\right|_{\Psi=\epsilon}=\sin \left(\frac{9 \pi}{4}\right) g(\epsilon, \theta, z) d \theta+\cos \left(\frac{9 \pi}{4}\right) d z=\left.\frac{\sqrt{2}}{2} \alpha\right|_{\Psi=\epsilon},
$$

and these have the same kernel. Furthermore, this modified structure contains the vector field $X=\nabla \Psi$, since $X$ points in the $-d / d \Psi$ direction. Finally, one can easily show that a perturbation of a constant- $z$ disc has a limit cycle in the characteristic foliation near $c=\epsilon / 2(c f$. [2]); hence, this defines an overtwisted structure containing $X$. This construction can obviously be done in the $C^{\infty}$ category using bump functions.

Example 5.8. Consider the flow on $S^{3}$ (considered as the unit sphere in $\mathbb{R}^{4}$ with the induced metric) given by the gradient of the function

$$
\Psi=\frac{1}{2}\left(x_{1}^{2}+x_{2}^{2}\right)-\frac{1}{2}\left(x_{3}^{2}+x_{4}^{2}\right)
$$

the gradient being taken in $S^{3}$. One can check that the fixed point set consists of a pair of unknots linked once in a Hopf link, as in Figure 6. The standard tight contact form on $S^{3}$ is

$$
\alpha=\frac{1}{2}\left(x_{1} d x_{2}-x_{2} d x_{1}+x_{3} d x_{4}-x_{4} d x_{3}\right) .
$$

A simple calculation shows that $\alpha$ is a contact form on $S^{3}$ with $\nabla \Psi \subset \operatorname{ker} \alpha$. However, we may Lutz twist this structure in a neighborhood of the fixed point links: a family of such overtwisted forms $\left(n \in \mathbb{Z}^{+}\right)$is given by [10]

$$
\begin{aligned}
\alpha_{n} & =\cos \left(\frac{\pi}{4}+n \pi\left(x_{3}^{2}+x_{4}^{2}\right)\right)\left(x_{1} d x_{2}-x_{2} d x_{1}\right) \\
& +\sin \left(\frac{\pi}{4}+n \pi\left(x_{3}^{2}+x_{4}^{2}\right)\right)\left(x_{3} d x_{4}-x_{4} d x_{3}\right),
\end{aligned}
$$

from which it can be shown that $\nabla \Psi \subset \operatorname{ker} \alpha_{n}$. Here, the integer $n$ denotes the number of twists that the plane field undergoes as an orbit travels from source to sink in Figure 6.

\section{Two questions}

This work has focused on the case of gradient flows in plane fields in dimension three, as the round-handle theory is most interesting here. However, there are natural questions about gradient flows in arbitrary distributions for manifolds of dimension greater than three. We do not present any results in this area, but 


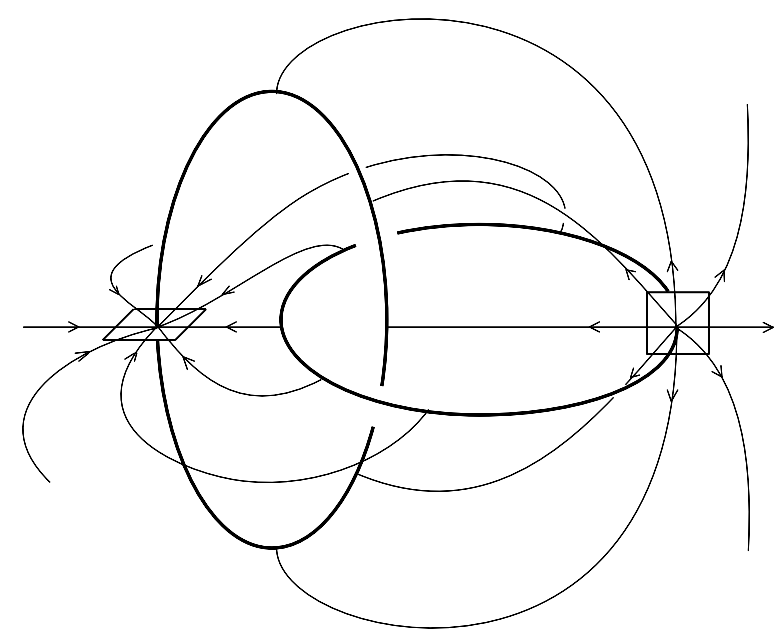

Figure 6.

The gradient field on $S^{3}$ having a Hopf link of fixed points exists within both tight and overtwisted contact structures.

rather note that many of the tools remain valid: fixed point sets of a vector field constrained to a codimension- $k$ distribution consists of a finite collection of embedded $k$-dimensional submanifolds.

Two problems emerge. In the case of a codimension-one distribution, nondegenerate gradient fields induce round-handle decompositions. However, every manifold of dimension greater than three whose Euler characteristic is zero possesses an RHD. Are there any such manifolds of dimension greater than three which do not possess a nondegenerate gradient field tangent to a codimension-one distribution? Secondly, in the case of higher codimension distributions, what restrictions exist on the topology of the fixed point sets? The case of a plane field on a four-manifold is particularly interesting with respect to the genera of the (two-dimensional) fixed point sets.

\section{Acknowledgments}

This work has been supported in part by the National Science Foundation [JE: grant DMS-9705949; RG: grant DMS-9508846]. The authors wish to thank Mark Brittenham, John Franks, Will Kazez, Alec Norton, and Todd Young for their input. Special thanks are due the referee for constructive remarks.

\section{References}

[1] D. Asimov, Round handles and non-singular Morse-Smale flows. Ann. Math. 102 (1975), 
$41-54$.

[2] D. Bennequin, Entrelacements et équations de Pfaff. Asterisque 107-108 (1983) 87-161.

[3] J. Casasayas, J. Martinez Alfaro and A. Nunes, Knotted periodic orbits and integrability. In: Hamiltonian Systems and Celestial Mechanics (Guanajuato 1991), 4, Adv. Ser. Nonlinear Dynam., pp 35-44. World Sci. Pub., River Edge, NJ, 1993.

[4] D. Eisenbud, U. Hirsch, and W. Neumann, Transverse foliations of Seifert bundles and self-homeomorphisms of the circle. Comment. Math. Helvetici 56 (1981), 638-660.

[5] Y. Eliashberg, Classification of overtwisted contact structures on 3-manifolds. Invent. Math. 98 (1989), 623-637.

[6] Y. Eliashberg, Contact 3-manifolds twenty years since J. Martinet's work, Ann. Inst. Fourier, Grenoble 42 (1-2) (1992), 165-192.

[7] Y. Eliashberg and W. Thurston, Confoliations, volume 13 of University Lecture Series, American Mathematical Society, 1998.

[8] R. Ghrist, 'Chaotic' knots and 'wild' dynamics. Chaos, Solitons, and Fractoals 9(4-5) (1998), 583-598.

[9] C. Godbillon, Feuilletages: Études géométriques. Number 98 in Progress in Mathematics. Birkhäuser, 1991.

[10] J. Gonzalo and F. Varela, Modèles globaux des variétés de contact. Astérisque 107-108 (1983), 163-168.

[11] M. Grayson and C. Pugh, Critical sets in 3-space. IHES Publ. Math. 77 (1993), 5-61.

[12] J. Guckenheimer and P. Holmes, Nonlinear Oscillations, Dynamical Systems, and Bifurcations of Vector Fields. Springer-Verlag, New York 1983.

[13] M. Hirsch, Differential Topology. Springer-Verlag, 1976.

[14] M. Hirsch, C. Pugh, and M. Shub, Invariant Manifolds, volume 583 of Springer Lecture Notes in Mathematics, Springer-Verlag, Berlin, Heidelberg, New York 1970.

[15] D. Koditschek and E. Rimon, Robot navigation functions on manifolds with boundary. Adv. in Appl. Math. 11(4) (1990), 412-442.

[16] R. Lutz, Structures de contact sur les fibrés principaux en cercles de dimension trois. Ann. Inst. Fourier 3 (1977), 1-15.

[17] J. Martinet, Formes de contact sur les variétés de dimension 3, pp. 142-163. Number 209 in Springer Lecture Notes in Math., Springer-Verlag, 1971.

[18] J. Morgan, Nonsingular Morse-Smale flows on 3-dimensional manifolds. Topology 18 (1978), $41-54$.

[19] S. Novikov, Topology of foliations. Trans. Moscow Math. Soc. 14 (1967), 268-304.

[20] R. Sacksteder, Limit sets of foliations. Am. J. Math. 87 (1965), 79-102.

[21] D. Tischler, On fibering certain foliated manifolds over $S^{1}$. Topology 9 (1970), 153-154.

[22] M. Wada, Closed orbits of nonsingular Morse-Smale flows on $S^{3}$. J. Math. Soc. Japan 41(3) (1989), 405-413.

John Etnyre

Department of Mathematics

Stanford University

Stanford, CA 94305

USA

(Received: December 9, 1997)
Robert Ghrist

School of Mathematics

Georgia Institute of Technology

Atlanta, GA 30332-0160

USA

e-mail: ghrist@math.gatech.edu 